Renata K. Batista Coelho Deus ${ }^{1}$ Alcibíades Bustamante ${ }^{1,2}$ Vítor Pires Lopes ${ }^{3}$ André F. Teixeira e Seabra ${ }^{1}$ Rui Manuel Garganta da Silva ${ }^{1}$ José António Ribeiro Maia ${ }^{1}$

\title{
COORDENAÇÃO MOTORA: ESTUDO DE TRACKING EM CRIANÇAS DOS 6 AOS 10 ANOS DA REGIÃO AUTÔNOMA DOS AÇORES, PORTUGAL
}

\author{
MOTOR COORDINATION: STUDYING TRACKING IN 6 TO 10-YEAR-OLD \\ CHILDREN IN THE AZORES AUTONOMOUS REGION, PORTUGAL
}

\begin{abstract}
RESUMO
O objetivo do estudo foi caracterizar o desenvolvimento coordenativo em crianças açorianas dos 6 aos 10 anos em função do sexo e estimar a estabilidade da mudança intra-individual nas diferenças inter-individuais ao longo dos quatro anos. A amostra de 285 crianças (143 meninos e 142 meninas) é proveniente do estudo longitudinal-misto da Região Autônoma dos Açores. A coordenação motora foi determinada com a bateria de testes KTK que compreende 4 provas: Equilíbrio à retaguarda, Saltos laterais, Saltos monopedais e Transposição lateral. As estatísticas descritivas básicas, a ANOVA II, assim como as auto-correlações foram calculados no SPSS 15. Recorremos ao $\gamma$ de Foulkes e Davies para verificar a quantidade de crianças que mantêm a mesma posição relativa. Este cálculo foi realizado no software LDA. As medidas descritivas básicas revelaram um incremento das médias, em ambos os sexos, ao longo da idade. A ANOVA II mostrou diferenças significativas em todos os testes ao longo dos quatro anos e, somente no teste Equilíbrio à Retaguarda foi encontrada uma interação significativa entre tempo e sexo. A correlação de Pearson apresentou uma estabilidade moderada. No teste Equilíbrio à Retaguarda, em ambos os sexos, e Saltos Monopedais nos meninos não se verificou nenhum tracking no desempenho o que revela uma forte heterogeneidade no desenvolvimento da Coordenação Motora ao longo dos quatro anos. Tais resultados reafirmam o direito à diferença nos níveis de coordenação e apelam para o entendimento do seu significado em termos pedagógicos.
\end{abstract}

Palavras-chave: Coordenação; Longitudinal; Estabilidade; Tracking; Crianças.

\begin{abstract}
The aims of the present study were to describe the development of motor coordination of children from the Azores aged 6 to 10 years of age of both genders; and to estimate the stability of intra-individual changes in inter-individual differences over 4 years. The sample comprised 285 children (143 boys and 142 girls) from the mixed-longitudinal study of the Azores Autonomous Region. Motor coordination was assessed with the KTK test battery which comprises 4 tests: Backward balance, Jumping sideways, Hopping on one leg and Shifting platforms. Basic descriptive statistics, ANOVA II, and auto-correlations were calculated in SPSS 15. We used the $\gamma$ of Foulkes and Davies to verify the proportion of children who maintained the same relative position. This calculation was performed using the software LDA. We found increases in mean values, in both genders, across the ages. ANOVA II showed significant differences in all tests over the 4 years, and a significant interaction between time and sex was only observed for the Backward balance. Auto-correlations presented moderate stability. Neither gender exhibited tracking in their performance in the Backward balance and the boys failed to do so in Hopping either, which demonstrates a strong heterogeneity in Motor Coordination development over the 4 years. These results show that children differ substantially along their motor development and calls for a clear understanding of its meaning in educational and didactic terms.
\end{abstract}

Key words: Coordination; Longitudinal; Stability; Tracking; Children.

1 Laboratório de Cineantropometria e Gabinete de Estatística Aplicada. Faculdade de Desporto. Universidade do Porto, Portugal. 2 Universidade Nacional de Educación “Enrique Guzmán y Valle”, La Cantuta. Lima, Perú.

3 Instituto Politécnico de Bragança, Portugal.I 


\section{INTRODUÇÃO}

Segundo Kiphard ${ }^{1}$, a coordenação é a interação harmoniosa e econômica do sistema musculoesquelético, do sistema nervoso e do sistema sensorial com a finalidade de produzir ações motoras precisas e equilibradas. A expressão multifacetada da capacidade de coordenação motora (CoM) é relevante no desenvolvimento do processo educativo da criança, não somente em termos psico-motores mas também no domínio do desempenho cognitivo². Estudar a CoM de crianças em idade escolar é também importante pela sua associação íntima a aspectos da aprendizagem motora e controle motor nas mais diversas tarefas do seu quotidiano e rotinas de vida, bem como pelas implicações pedagógicas e educativas que decorrem das aulas de Educação Física ${ }^{3}$.

De fato, é comum, para quem leciona ou treina crianças e adolescentes nas fases iniciais da sua carreira esportiva, identificar alguma debilidade ou insuficiência no seu rendimento ${ }^{4}$. A insuficiência da CoM remete a uma instabilidade motora geral, que envolve a qualidade do seu processo que pode ser atribuído a uma pobre interação das funções senso-neuro-musculares ${ }^{1}$ sendo de esperar que a Educação Física Escolar (EFE), dentro das suas atribuições fundamentais, possa identificar e corrigir essas insuficiências ${ }^{5}$.

Ao analisar minuciosamente diferentes aspectos dos níveis e padrões do desenvolvimento da CoM somos confrontados, com a necessidade de interpretar adequadamente a estabilidade e mudança que ocorrem em diferentes manifestações da CoM de crianças e adolescentes ao longo da sua escolaridade ${ }^{6}$. Ressalta daqui, claramente, que tal análise e interpretação exige informação longitudinal, supondo que os momentos de registro dessas características sejam equidistantes no tempo e, que forneçam explicações acerca do modo como ocorrem, ou não, a mudança intra-individual e as diferenças inter-individuais ${ }^{6}$.

O termo tracking expressa a ideia de manutenção de posição relativa no seio de uma distribuição de valores que se altera no tempo ${ }^{7}$. Estabilidade, mudança e previsão são aspectos essenciais do tracking ${ }^{4}$. Os primeiros autores a estudar o tracking, de modo sistemático e metodológico, e a sugerirem duas correntes fundamentais de pensamento metodológico foram Foulkes e Davies em 1981. Tracking pretende descrever um padrão acerca do crescimento ou mudança de um conjunto de padrões de crescimento ${ }^{5}$. Por não haver uma definição universal para o termo, o seu conteúdo apresenta a noção genérica da tendência de um indivíduo ou uma série deles, permanecerem num dado curso ou canal de crescimento ou mudança, refletindo estabilidade no seu padrão de mudança $a^{7,6,8}$.

A mudança pode ser identificada sistematicamente de dois modos distintos: a mudança a curto prazo, designada de variabilidade intra-individual que não representa uma verdadeira mudança na característica em causa e a mudança a longo prazo, relativamente irreversível, conhecida como mudança intra-individual. Estas facetas distintas da estabilidade e mudança refletem aspectos distintos das alterações que ocorrem no desenvolvimento de habilidades e aptidões motoras de crianças e jovens que reclamam uma identificação precisa por parte dos professores de Educação Física para justificarem a estrutura das suas opções didáticometodológica. Não se trata somente de perceber aspectos do desenvolvimento normativo ou modal, mas sobretudo a magnitude e significados das diferenças entre crianças e jovens ${ }^{7}$.

Alguns estudos referentes à mudança e estabilidade da CoM estão baseados em análises dos valores médios do desempenho em diferentes momentos da avaliação. Esta abordagem nos parece insuficiente, porque não permite leituras mais esclarecedoras sobre toda a informação decorrente de estudos longitudinais ${ }^{7}$. Lopes ${ }^{5}$, analisou, ao longo de um ano, os efeitos de diferentes programas de aulas de Educação Física no desenvolvimento dos níveis de CoM em crianças de 9 anos. Os resultados indicaram diferenças significativas nos testes $\mathrm{ER}[(\mathrm{F}=$ $11,879, p=0,001)$ e TL $(F=5,398, p=0,023)]$ na $1^{\text {a }}$ e $2^{a}$ avaliação e, SM [(F = 10,587, $\left.\left.p=0,002\right)\right]$ entre $1^{a}$, $2^{\mathrm{a}}$ e $3^{\mathrm{a}}$ avaliação.

Pesquisas acerca do tracking da aptidão física, da atividade física, de habilidades motoras, e da performance esportiva tem sido de grande interesse para os investigadores da Ciências do Esporte e, estudados em áreas tão distintas como as Ciências do Esporte, a Psicologia Industrial e a Epidemiologia da Atividade Física ${ }^{6,7,9}$.

Os estudos sobre tracking da CoM são praticamente inexistentes. Só conseguimos localizar um. Willimczik ${ }^{10}$ em seu estudo longitudinal com crianças alemãs em idade escolar (6,7 aos 10,7 anos) encontrou valores elevados de auto-correlações entre o primeiro e o segundo momento da avaliação $(0,80$ $\leq r \leq 0,82)$ e o primeiro e os quatro momentos $(0,63 \leq$ $r \leq 0,82)$.

Face à quase inexistência de estudos sobre estabilidade do desenvolvimento coordenativo e a sua relevância em termos pedagógicos, a presente pesquisa com crianças açorianas do $1^{\circ} \mathrm{Ciclo}$ Ensino Básico dos 6 aos 10 anos tem os seguintes propósitos: (1) caracterizar, genericamente, o nível de desenvolvimento coordenativo ao longo dos quatro anos de avaliação e, (2) estimar a estabilidade do desenvolvimento coordenativo.

\section{PROCEDIMENTOS METODOLÓGICOS}

\section{Amostra}

A amostra é proveniente do estudo longitudinalmisto da Região Autônoma dos Açores (RAA), que foi genericamente desenhado para avaliar o crescimento somático, a CoM, a Atividade Física e a Aptidão Física de crianças e adolescentes no intervalo etário entre os 6 e 19 anos. A amostra está repartida por 4 ilhas (Faial, Pico, São Miguel e Terceira), sendo constituída por 4 coortes. Para o presente estudo foram consideradas 285 crianças (142 meninas e 143 meninos) da $1^{\text {a }}$ coorte sendo acompanhadas dos 6 aos 10 anos. 
Avaliação da Coordenação Motora

Para avaliar a coordenação motora foi utilizada a bateria KTK (Körperkoordinationstest für Kinder - KTK) que foi desenvolvida, em 1974, pelos pesquisadores alemães Kiphard e Schilling ${ }^{11}$ considerando uma abordagem simultaneamente clínica e pedagógica. A estrutura final bem como o número de testes foram determinados com base em diversos estudos empíricos submetidos a procedimentos de análise fatorial. $\mathrm{O}$ objetivo principal da bateria era, e ainda continua a ser, o de identificar crianças com insuficiência no desenvolvimento coordenativo. Esta bateria é constituída por 4 testes: (1) equilíbrio à retaguarda (ER); (2) saltos laterais (SL); (3) saltos monopedais (SM); e (4) transposição lateral (TL). Neste estudo, os resultados das quatro provas serão analisados individualmente por forma de obter uma visão mais detalhada do desempenho coordenativo.

\section{Procedimentos Estatísticos}

Foi realizada a análise exploratória de dados para verificar possíveis erros de entrada das informações, a presença de outliers e a normalidade das distribuições. Para cálculo das estatísticas básicas, ANOVA II, valores de correlação $(r)$ e representação gráfica dos diagramas de dispersão utilizaram-se os programas informáticos SPSS 15, SYSTAT 10 e Microsoft EXCEL. Recorremos ao programa estatístico Longitudinal Data Analysis (LDA), especificamente à estatística Gama $(\gamma)$ de Foulkes e Davies para analisar o tracking. $\mathrm{O} \gamma$ é uma estatística descritiva do tracking que calcula a probabilidade de duas curvas ou perfis aleatórios de desempenho não se entrecruzarem, implicando um "esforço" de ajustamento dos valores a uma função linear ou quadrática. $O$ tracking será perfeito quando a posição relativa de um indivíduo for mantida no seio da distribuição durante os quatro pontos do tempo. $O$ cálculo do $\gamma$ de Foulkes e Davies exige um ajustamento prévio de cada trajetória individual, para que, em cada ponto do tempo, a distribuição dos valores de todos os sujeitos seja normal.

\section{Fiabilidade dos Dados}

Foi realizado um estudo piloto pelas equipes de investigação, garantindo que a amostra testada tivesse crianças de ambos os sexos pertencentes à primeira coorte do estudo. A correlação intra-classe $(R)$ foi calculada e seus resultados estão no Quadro 1.
Tabela 1- Valores do coeficiente de correlação intraclasse $(R)$.

\begin{tabular}{ccccc}
\hline \multirow{2}{*}{$\begin{array}{c}\text { variáveis } \\
\text { coordenação } \\
\text { motora }\end{array}$} & $\begin{array}{c}\text { múmero } \\
\text { de alunos }\end{array}$ & $\begin{array}{c}\text { valor } \\
\text { de R }\end{array}$ & $\begin{array}{c}\text { múmero } \\
\text { de alunos }\end{array}$ & $\begin{array}{c}\text { valor } \\
\text { de R }\end{array}$ \\
\hline ER & 13 & 0,81 & 12 & 0,79 \\
SL & 13 & 0,91 & 12 & 0,83 \\
SM & 13 & 0,87 & 12 & 0,87 \\
TL & 13 & 0,75 & 12 & 0,91 \\
\hline
\end{tabular}

\section{RESULTADOS}

Os resultados serão apresentados sequencialmente: em primeiro lugar lidaremos com as estatísticas descritivas, suas respectivas representações gráficas e testes formais das mudanças das médias; em seguida abordaremos o tracking com base em procedimentos estatísticos de correlação de Pearson e $\gamma$ de Foulkes e Davies.

O Quadro 2 e Figura 1 apresentam os aspectos descritivos fundamentais das quatro provas (média, desvio padrão, valor mínimo e máximo). Tal como esperado, em ambos os sexos, a maioria dos testes, as médias revelaram um aumento significativo ao longo da idade. Os resultados da ANOVA II revelou diferenças significativas em todos os testes ao longo dos quatro anos, em ambos os sexos ( $T L, F=270,063$; $p<0,001$; $S M, F=270,866 ; p<0,001 ; S L, F=447,199 ; p<0,001$; $E R, F=148,936 ; p<0,001)$. Somente no teste ER se verificou uma interação estatisticamente significativa entre o tempo e o sexo.

A magnitude dos desvios-padrão que indica, genericamente, a variação dos valores individuais em torno da média, revela diferenças inter-individuais marcantes em cada teste, sobretudo nos testes ER, SM e SL. O teste TL é o que apresenta menor variação nos valores do desvio-padrão em ambos os sexos.

O tabela 3 apresenta a forma mais simples de análise do tracking - auto-correlações de cada um dos testes nos quatro momentos. Na Figura 2 está a representação gráfica do comportamento de cada prova, em cada sexo, ao longo da idade. Os resultados são baixos-a-moderados ao longo do tempo, revelando uma estabilidade moderada. Os valores mais reduzidos foram encontrados nas meninas, entre a $1^{\mathrm{a}}$ e $3^{\mathrm{a}}$ avaliação nos testes ER $(r=0,27)$ e TL $(r=0,32)$ e, entre a $1^{a}$ e $4^{a}$ avaliação nos testes TL $(r=0,36)$ e SM $(r=0,15)$. Nos meninos, os valores mais baixos situam-se entre a $1^{\mathrm{a}}$ e $3^{\mathrm{a}}$

Tabela 2- Valores médios, ( \pm ) desvios-padrão, valores mínimos e máximos dos quatro itens da bateria KTK em cada momento da avaliação.

\begin{tabular}{|c|c|c|c|c|c|c|c|c|}
\hline \multirow[b]{2}{*}{ FEM } & \multicolumn{2}{|c|}{$1^{a}$ avaliação } & \multicolumn{2}{|c|}{$2^{a}$ avaliação } & \multicolumn{2}{|c|}{$3^{a}$ avaliação } & \multicolumn{2}{|c|}{$4^{a}$ avaliação } \\
\hline & $M \pm d p$ & min-máx & $M \pm d p$ & min-máx & $\mathrm{M} \pm \mathrm{dp}$ & min-máx & $M \pm d p$ & min-máx \\
\hline ER & $28,35 \pm 14,81$ & $1-69$ & $36,07 \pm 14,18$ & $8-72$ & $40,18 \pm 11,78$ & $9-66$ & $46,15 \pm 13,78$ & $13-72$ \\
\hline SM & $14,58 \pm 9,90$ & $0-51$ & $22,99 \pm 13,41$ & $1-70$ & $34,67 \pm 17,13$ & $0-71$ & $39,43 \pm 18,62$ & $0-72$ \\
\hline SL & $28,30 \pm 8,73$ & $10-62$ & $35,67 \pm 10,03$ & $16-64$ & $42,99 \pm 13,76$ & $11-75$ & $52,44 \pm 12,11$ & $18-78$ \\
\hline $\mathrm{TL}$ & $13,87 \pm 2,53$ & $6-20$ & $17,89 \pm 3,19$ & $8-27$ & $19,74 \pm 3,81$ & $11-32$ & $20,16 \pm 3,43$ & $12-30$ \\
\hline \multicolumn{9}{|c|}{ MASC } \\
\hline ER & $29,20 \pm 14,20$ & $3-69$ & $37,06 \pm 14,08$ & $8-68$ & $42,43 \pm 13,91$ & $9-72$ & $48,22 \pm 13,09$ & $14-72$ \\
\hline SM & $37,06 \pm 14,08$ & $8-68$ & $27,18 \pm 15,70$ & $3-72$ & $37,22 \pm 17,99$ & $0-72$ & $41,11 \pm 19,18$ & $3-72$ \\
\hline SL & $31,34 \pm 9,73$ & $8-60$ & $36,90 \pm 10,84$ & $9-75$ & $43,94 \pm 10,07$ & $18-82$ & $53,63 \pm 13,89$ & $15-91$ \\
\hline TL & $15,07 \pm 2,68$ & $8-21$ & $18,38 \pm 3,26$ & $10-28$ & $20,68 \pm 3,40$ & $13-34$ & $20,92 \pm 3,74$ & $11-33$ \\
\hline
\end{tabular}



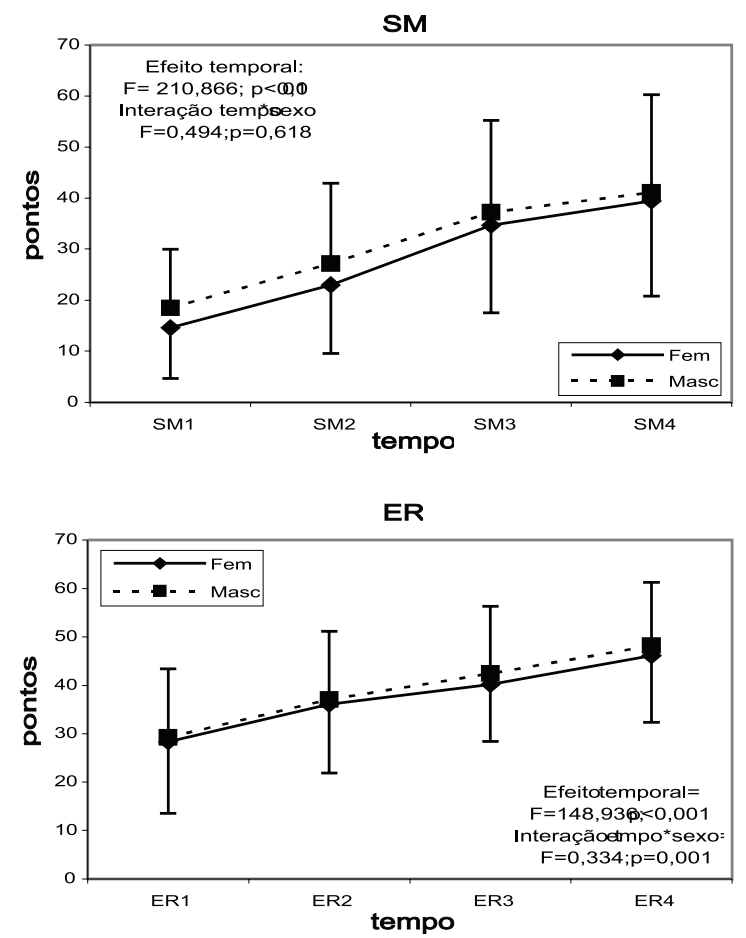

TL

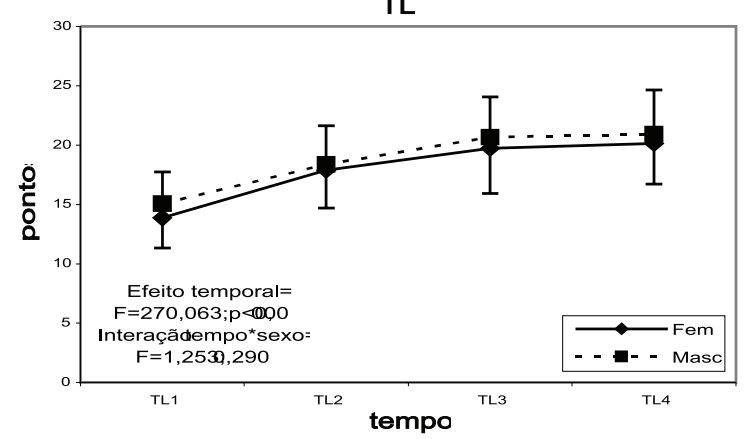

SL

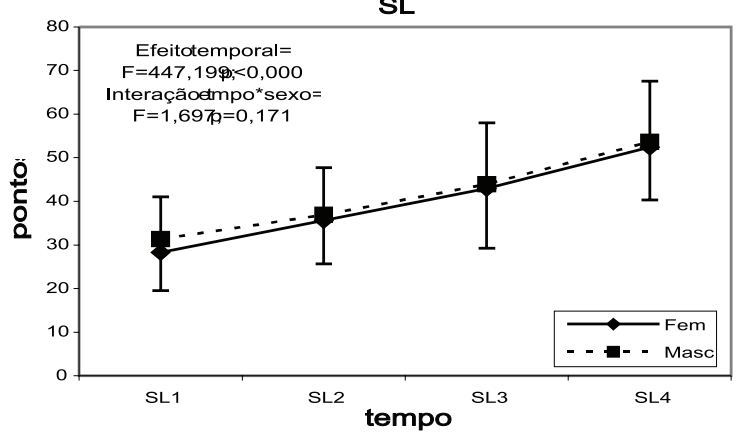

\begin{tabular}{cccccccccc}
\hline & TL1 & TL2 & TL3 & TL4 & & SL1 & SL2 & SL3 & SL4 \\
\hline Fem & 13,87 & 17,89 & 19,74 & 20,16 & Fem & 28,30 & 35,67 & 42,99 & 52,44 \\
Masc & 15,07 & 18,38 & 20,68 & 20,92 & Masc & 31,34 & 36,90 & 43,94 & 53,63 \\
\hline & & & & & & & & \\
\hline & SM1 & SM2 & SM3 & SM4 & & ER1 & ER2 & ER3 & ER4 \\
\hline Fem & 14,58 & 22,99 & 34,67 & 39,43 & Fem & 28,35 & 36,07 & 40,18 & 46,15 \\
Masc & 18,50 & 27,18 & 37,22 & 41,11 & Masc & 29,20 & 37,06 & 42,43 & 48,22 \\
\hline
\end{tabular}

Figura 1- Comportamento das médias ( \pm desvio padrão) de cada teste da bateria KTK ao longo dos quatro momentos da avaliação em ambos os sexos.

Tabela 3 - Matrizes de auto-correlação entre os diferentes momentos de avaliação

\begin{tabular}{|c|c|c|c|c|c|c|}
\hline \multicolumn{7}{|l|}{$\begin{array}{l}\text { Correlação de } \\
\text { Pearson (R) }\end{array}$} \\
\hline \multicolumn{7}{|l|}{ ER } \\
\hline $2^{\mathrm{a}}$ avaliação & 0.541 & & & 0.414 & & \\
\hline $3^{a}$ avaliação & 0.274 & 0.443 & & 0.274 & 0.580 & \\
\hline $4^{\mathrm{a}}$ avaliação & 0.369 & 0.585 & 0.538 & 0.347 & 0.656 & 0.508 \\
\hline \multicolumn{7}{|l|}{$\overline{\mathrm{SL}}$} \\
\hline $2^{a}$ avaliação & 0.569 & & & 0.741 & & \\
\hline $3^{a}$ avaliação & 0.517 & 0.523 & & 0.604 & 0.647 & \\
\hline $4^{a}$ avaliação & 0.586 & 0.546 & 0.627 & 0.648 & 0.648 & 0.680 \\
\hline \multicolumn{7}{|l|}{$\mathrm{TL}$} \\
\hline $2^{a}$ avaliação & 0.443 & & & 0.515 & & \\
\hline $3^{a}$ avaliação & 0.321 & 0.333 & & 0.168 & 0.282 & \\
\hline $4^{a}$ avaliação & 0.360 & 0.321 & 0.281 & 0.348 & 0.492 & 0.378 \\
\hline \multicolumn{7}{|l|}{ SM } \\
\hline $2^{a}$ avaliação & 0.742 & & & 0.727 & & \\
\hline $3^{a}$ avaliação & 0.650 & 0.704 & & 0.662 & 0.723 & \\
\hline $4^{\mathrm{a}}$ avaliação & 0.155 & 0.195 & 0.369 & 0.387 & 0.395 & 0.503 \\
\hline
\end{tabular}

avaliação nos testes $\operatorname{ER}(r=0,27)$ e TL $(r=0,17)$ e, entre a $1^{a}$ e a $4^{a}$ avaliação TL $(r=0,35)$ e SM $(r=0,39)$. Os valores mais elevados, acima de $r=0,70$, foram obtidos no teste SM nas meninas e SL e SM nos meninos.

A Figura 3 mostra as trajetórias intra-individuais e as diferenças entre indivíduos no seio de cada 

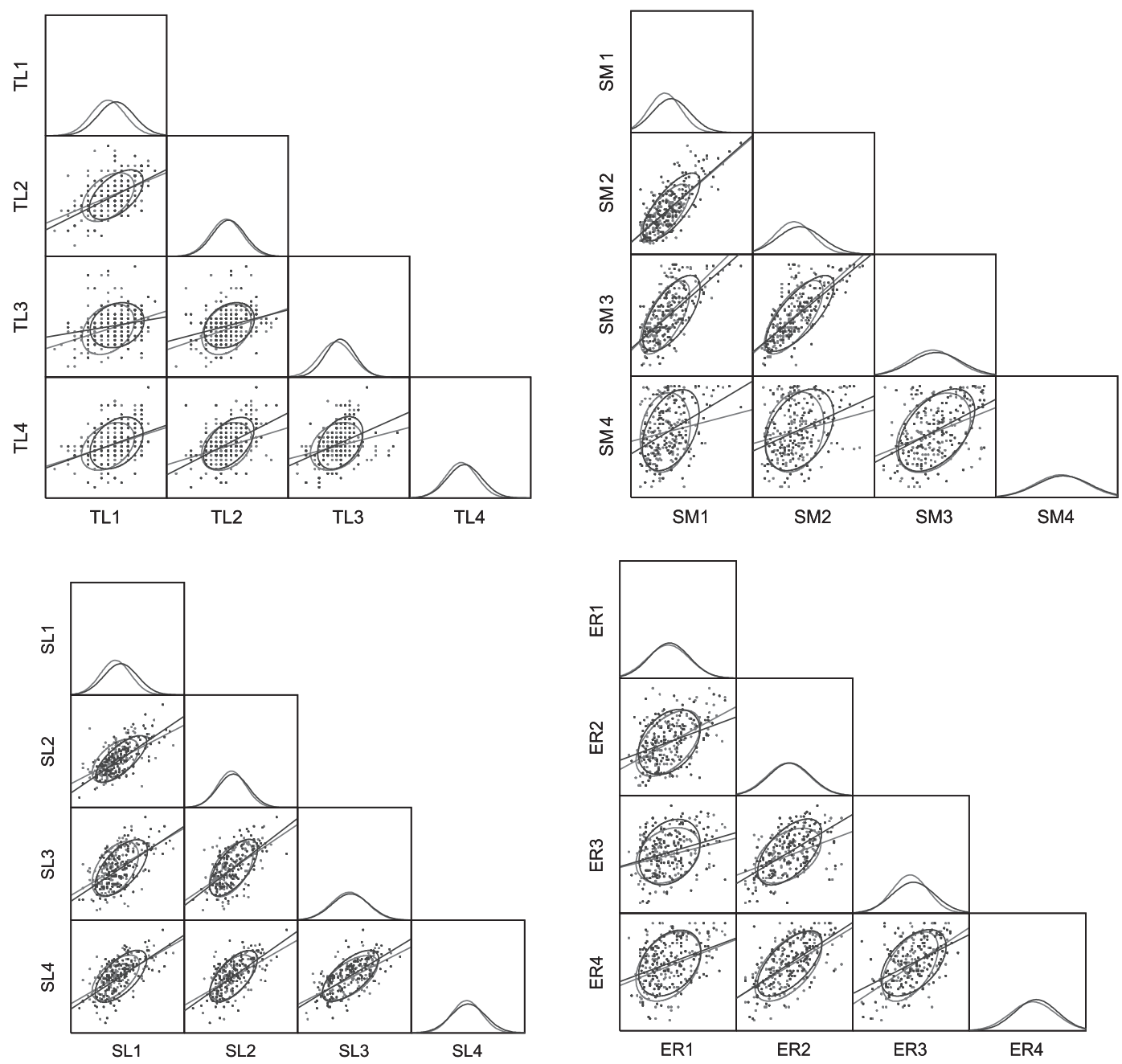

Figura 2- Diagramas de dispersão entre os 4 momentos de avaliação. (Os gráficos incluem retas de regressão para cada sexo, elipses que contêm os pontos de cada sujeito no tempo e polígonos de frequência para mostrar a normalidade das distribuições).

sexo ao longo de quatro anos de estudo em cada uma das provas do KTK. É mais do que evidente destes "sparghetiplots", a presença de elevada heterogeneidade na mudança intra-individual bem como nas diferenças de desempenho coordenativo, ao longo do tempo, nas meninas e nos meninos.

O Quadro 4 apresenta os valores de estabilidade em cada teste, a partir do $\gamma$ de Foulkes e Davies. De acordo com os resultados referidos anteriormente, $0 \gamma$ evidenciou valores onde não se verifica a presença de tracking nos testes ER em ambos os sexos $(\gamma=0,54 \pm$ $0,01)$, e no teste SM nos meninos $(\gamma=0,54 \pm 0,01)$. O tracking é irrelevante nos testes $S L$ em ambos os sexos $(\gamma=0,43 \pm 0,01)$, SM nas meninas $(\gamma=0,29 \pm 0,01)$, e TL nos meninos $(\gamma=0,44 \pm 0,01)$ e nas meninas $(\gamma=$ $0,41 \pm 0,01)$.

\section{DISCUSSÃO}

O presente estudo aporta informações sobre o nível de desenvolvimento coordenativo de crianças açorianas dos 6 aos 10 anos, assim como valores da sua estabilidade ao longo dos quatro anos de avaliação.
Quadro 4 - Valores do Índice de Foulkes e Davies ( \pm erropadrão) e intervalo de confiança.

\begin{tabular}{|c|c|c|c|c|}
\hline & \multirow{2}{*}{$\begin{array}{c}\text { Meninos } \\
\gamma \pm \mathrm{E \Pi}\end{array}$} & \multicolumn{3}{|c|}{ Meninas } \\
\hline & & IC 95\% & $\gamma \pm$ ЕП & IC 95\% \\
\hline ER & 0,54 & $0,51-0,57$ & 0,54 & $0,51-0,57$ \\
\hline SL & 0,43 & $0,40-0,45$ & 0,43 & $0,40-0,45$ \\
\hline SM & 0,54 & $0,51-0,57$ & 0,29 & $0,27-0,32$ \\
\hline TL & 0,54 & $0,40-0,47$ & 0,41 & $0,41-0,44$ \\
\hline
\end{tabular}

Os resultados das estimativas de fiabilidade, muito semelhantes aos encontrados em outros estudos ${ }^{5,12,13}$, indicam uma qualidade elevada da informação, permitindo, portanto, que as inferências estatísticas sejam consistentes.

Os resultados da análise descritiva (Quadro 2) confirmam que ao longo dos quatro anos existe uma tendência clara para incrementar os valores médios na maioria das provas do KTK em ambos os sexos. Este é um comportamento esperado, não só a CoM, mas também para as mais diversas aptidões face ao desenvolvimento motor que ocorre ao longo dos quatro anos $^{3,14}$. Resultados semelhantes aos do presente estudo foram encontrados por Kiphard e Schilling ${ }^{13}$, 
TL
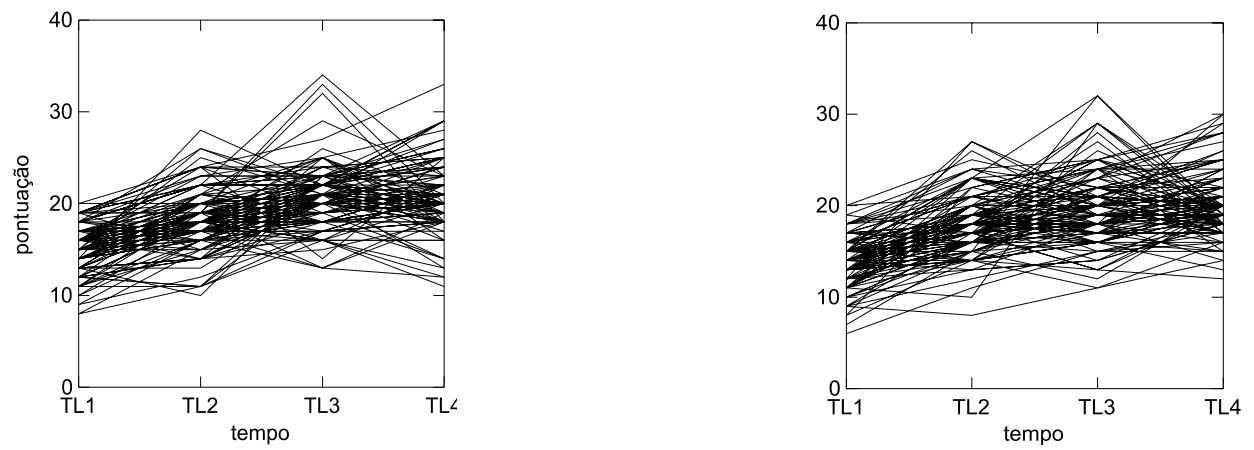

SM
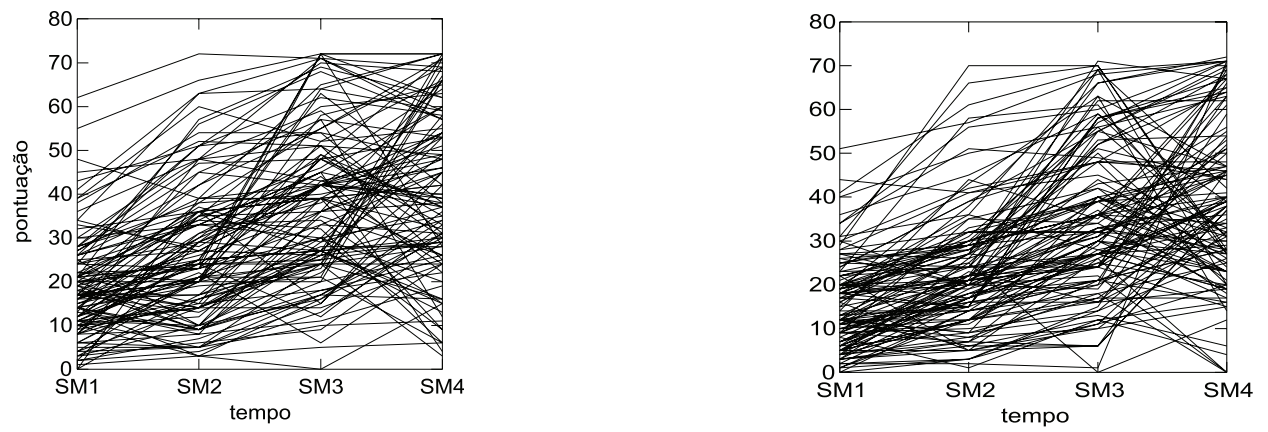

SL
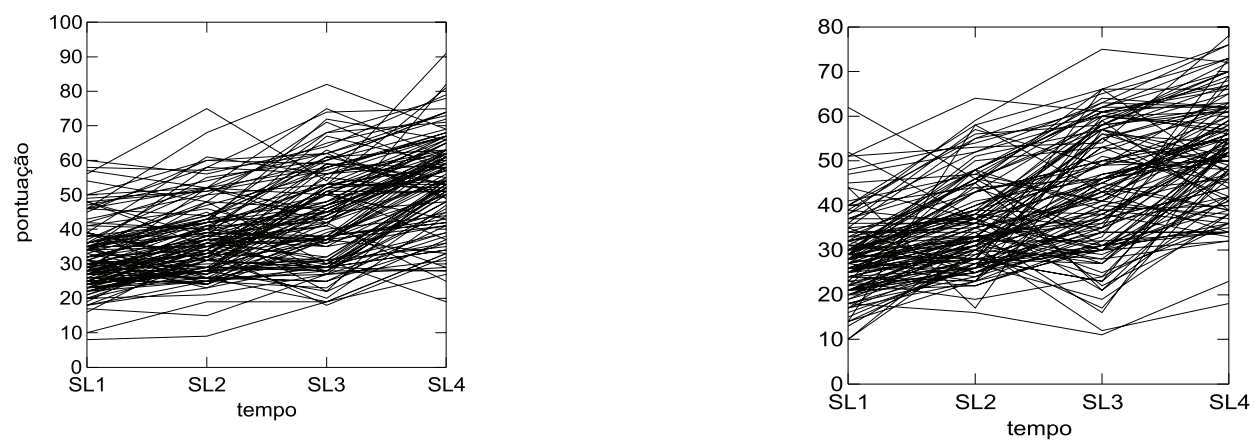

ER
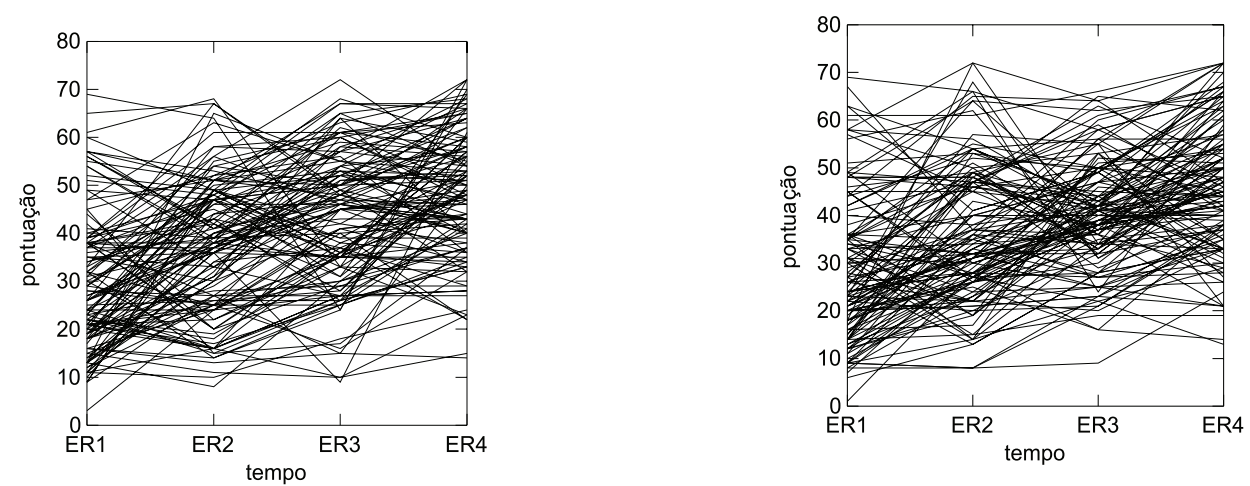

Figura 3 - Trajetórias intra-individuais e diferenças entre indivíduos nos diferentes testes do KTK ao longo dos quarto anos de estudo. As figuras da esquerda referem-se aos meninos e da direita às meninas.

Willimczik ${ }^{10}$ e Lopes $^{5}$. Em todos os testes as meninas apresentam valores inferiores aos meninos. Também Willimczik ${ }^{10}$ verificou este dimorfismo sexual, embora nas meninas, e apenas aos 6 anos, houvesse uma vantagem no desempenho coordenativo.

Os valores dos desvios-padrão são de magnitude elevada no teste SM em ambos os sexos, existindo uma tendência de incremento progressivo nos quatro 
momentos de avaliação (9,90 a 18,62 e 11,47 a 19,18 nas meninas e meninos, respectivamente). Estes resultados traduzem um incremento na variabilidade inter-individual, refletindo trajetórias distintas de desenvolvimento da CoM entre as crianças ao longo dos quatro anos. No teste TL, a magnitude dos valores é reduzida (2,53 a 3,43 e 2,68 a 3,74 nas meninas e meninos, respectivamente), salientando a manutenção da variabilidade inter-individual. A heterogeneidade encontrada nos valores do desvio-padrão do teste SM, em função da idade, reclama uma atenção elevada no planeamento das aulas de Educação Física, especialmente a sua estrutura didática-pedagógica em cada ano de escolaridade ${ }^{5}$.

A estabilidade ou tracking da CoM foi avaliada inicialmente com base nos procedimentos de autocorrelação. Malina ${ }^{8}$ descreve, de modo impressionista, a qualificação a atribuir nos resultados do tracking com base nas auto-correlações sugerindo que uma correlação inferior a 0,30 seja considerada baixa, entre 0,30 e 0,60 moderada e maior que 0,60 de estabilidade elevada. As auto-correlações (tabela 3) entre as avaliações das componentes da bateria KTK são na sua maioria moderadas; a mais baixa, e a mais alta foram encontradas nas meninas no teste SM $(0,15 \leq r \leq 0,74)$. Willimczik ${ }^{10}$ em seu estudo encontrou valores moderados-a-elevados nas correlações $(0,63$ $\leq r \leq 0,83$ ). Nos diagramas de dispersão (Figura 2) podemos observar a concentração dos valores, de cada sexo, em torno da reta. Relativamente ao teste SL, a concentração destes valores em torno da reta é mais elevado e os pontos estão na maioria contidos numa elipse mais alongada, sugerindo uma maior semelhança intra-sexo. Willimczik ${ }^{10}$ em seu estudo, não encontrou diferenças significativas entre os sexos em nenhum momento da avaliação. Por sua vez no estudo de Lopes ${ }^{3}$ foram encontradas diferenças significativas no nível de desenvolvimento de coordenação motora apenas aos 8 e aos 9 anos de idade, onde os meninos apresentam níveis superiores às meninas. Em contraposição Kiphard e Shilling ${ }^{11}$ verificaram que as meninas tinham aproveitamentos superiores aos meninos dos 6 aos 10 anos. Esta diferença nos níveis de desenvolvimento da coordenação motora entre meninos e meninas pode estar relacionado com diferentes oportunidades da prática esportiva-motora, e de atividades físicas, salientando o papel social e as expectativas distintas associadas aos dois sexos, favorecendo os meninos.

As estatísticas referentes ao coeficiente de correlação de Pearson podem não ser a melhor forma para estimar o tracking, ainda que seja intuitivamente fácil de calcular e interpretar,13. A ausência de um modelo estrito para expressar a dinâmica da mudança intraindividual e as diferenças entre sujeitos, bem como uma definição precisa de manutenção da posição relativa implica o recurso a um outro procedimento mais esclarecedor. $\mathrm{O} \gamma$ Foulkes e Davies é uma estatística interessante para descrever a noção de tracking, dado examinar a quantidade de trajetórias de mudança das crianças que mantêm a mesma posição relativa em cada teste do KTK (i.e., cujas trajetórias intra-individuais que não se cruzam com outras em nenhum ponto do tempo). Foulkes e Davies ${ }^{7}$ sugerem que se o valor de $\gamma=0,50$ não se verifica qualquer tracking; se $\gamma=$ 1,00 o tracking é perfeito, e se $\gamma<0,50$, o tracking é irrelevante.

De acordo com os valores encontrados de $\gamma$, o tracking foi irrelevante nos testes SL $(\gamma=0,43 \pm 0,01)$ e $\operatorname{TL}(\gamma=0,44 \pm 0,01)$ nos meninos, e SL $(\gamma=0,43 \pm 0,01)$ e SM $(\gamma=0,29 \pm 0,01)$ nas meninas, ou seja, houve ausência de tracking ou canalização dos valores do desenvolvimento coordenativo ao longo dos quatro anos. No teste $\operatorname{ER}(\gamma=0,54 \pm 0,01)$, em ambos os sexos, e nos meninos no teste SM $(\gamma=0,54 \pm 0,01)$, não se verifica qualquer tracking no desempenho da CoM das crianças. O cruzamento das trajetórias inter-individuais em cada teste pode ser observado graficamente na Figura 3. Pode-se se verificar que no teste SM o cruzamento das trajetórias é mais elevado, indicando uma elevada variabilidade inter-individual nas mudanças que ocorrem na CoM das crianças dos dois sexos.

Apesar dos valores médios terem apresentado um incremento positivo ao longo dos quatro anos de estudo, a estabilidade foi baixa-a-moderada, evidenciando que existe uma elevada variabilidade na trajetória de cada criança. Isso significa que estamos diante de um comportamento instável, o que pode colocar algumas dificuldades no sentido de prever o desempenho individual ou do grupo e que reclamam uma atenção redobrada por parte dos professores de Educação Física. Esses valores podem também estar relacionados tanto com variações na velocidade de crescimento das crianças, bem como da diversidade das experiências e repertórios motores ${ }^{15}$.

Édeesperaraexistência dealgumaheterogeneidade no desenvolvimento do perfil coordenativo de cada criança. Ao mesmo tempo, e de acordo com posições normativistas, no seio de cada idade, as crianças deveriam apresentar um perfil de coordenação que as tornassem relativamente homogêneas, ou seja, para cada idade seria de esperar que uma qualquer criança deveria mostrar um nível de coordenação motora relativamente preciso $^{3}$. Como o desenvolvimento das capacidades coordenativas não depende somente dos processos de maturação biológica, mas da quantidade e qualidade de experiências motoras a que a criança estiver exposta ${ }^{15}$, uma das grandes preocupações educativas deveriam situam-se ao nível das aulas de Educação Física. Lopes ${ }^{5}$ analisou o efeito do treinoinstrução na mudança da CoM ao longo de um ano letivo. As crianças foram sujeitas a dois diferentes programas (alternativo e oficial) e frequências semanais (2 e 3 aulas/semanais). Cinco turmas de crianças de 9 anos foram repartidas em: quatro grupos experimentais e um de controle. A CoM foi avaliada através do KTK em 3 momentos distintos. Os resultados indicaram que a capacidade de coordenação corporal foi mais elevada nos grupos de crianças sujeitas as aulas de Educação Física (grupos experimentais) do que o grupo controle. As crianças sujeitas ao programa alternativo 
obteve ganhos superiores às crianças do grupo oficial $\mathrm{e}$, as crianças com três aulas semanais teve melhor prestação que as crianças com duas aulas semanais.

\section{CONCLUSÃO}

De acordo com os propósitos delineados para este estudo, podemos concluir que: (1) em todas as provas do KTK, em ambos os sexos e ao longo dos quatro anos, houve um incremento significativo dos valores médios; (2) a estabilidade ou o tracking foi moderado-a-baixo; esta circunstância revela uma forte heterogeneidade no desenvolvimento da CoM das crianças da RAA. Esta plasticidade no seu desenvolvimento deve ser cuidadosamente monitorizada e considerada no estabelecimento de objetivos educativos e na construção didáticometodológica das aulas. Os resultados vêm reafirmar o direito à diferença e reclamam o entendimento do seu significado em termos pedagógicos por todos os professores de Educação Física.

\section{REFERÊNCIAS BIBLIOGRÁFICAS}

1. Kiphard EJ. Insuficiencias de movimiento y de coordinación en la edad de la escuela primaria. Buenos Aires: Editorial Kapelusz, 1976.

2. Smits-Engelsman BCM, Henderson S, Michels C. The assessment of children with Developmental Coordination Disorders in the Netherlands: The relationship between the Movement Assessment Battery for Children and the Körperkoordinations Test für Kinder. Hum Mov Sci 1998;17:699-709.

3. Lopes VP, Maia JA, Silva R, Seabra A, Morais F. Estudo do nível de desenvolvimento da coordenação motora da população escolar (6 a 10 anos de idade) da Região Autónoma dos Açores. Rev Port Ciên Desporto 2003;3:47-60.

4. Maia JAR, Lopes VP. Crescimento e desenvolvimento de crianças e jovens Açorianos. O que pais, professores, pediatras e nutricionistas gostariam de saber. Porto: DREFD e FADEUP, 2007b.

5. Lopes VP. Análise dos Efeitos de dois programas distintos de Educação Física na expresso da aptidão física, coordenação e habilidades motoras em crianças do ensino primário. [Tese de doutorado]. Porto; FADEUP; 1997.

6. Lopes VP, Maia JAR, Silva RG, Seabra A, Vasques CMS. Estabilidade e Mudança nos níveis de Actividade Física. Uma revisão da literatura baseada na noção e valores do tracking. Rev. bras. cineantropom. desempenho hum 2005;7:76-86.

7. Maia JAR, Garganta RM, Seabra A, Lopes, VP, Silva S, Meira Jr. C. Explorando a noção e significado de Tracking. Um percurso didáctico para investigadores. [periódico on line]. 2007. Disponível em <URL: http:// www.psicologia.com.pt/artigos/textos/A0348.pdf> [2007 ago 14].

8. Malina RM. Adherence to physical activity from childhood to adulthood: A perspective from tracking studies. Quest 2001;53:346-355.

9. Maia JAR, Lefevre J, Claessens A, Renson R, Vanreusel $B$, Beunen G. Tracking of physical fitness during adolescence: a panel study in boys. Med Sci Spo Exercise 2000; (33):765-771.

10. Willimczik, K. Development of motor control capability (body coordination) of 6-to 10-year-old children: Results of a Longitudinal Study. In: Ostyn M., Beunen G, Simons J. (eds.). Kinanthropometry II. Baltimore: University Park Press, 1980. 328-346.

11. Kiphard, BJ, Schilling F. Körperkoordinations Test für Kinder. Beltz Test GmbH. Weinheim: 1974.

12. Bustamante A, Tavara I. Crecimiento somático, coordinación motora y actividad asociada a la salud de ninos del Nivel Primário del distrito de Lurigancho - Chosica. Lima, 2004.

13. Bustamante A, Caballero L, Quispe S, Rodríguez G, Enciso N, Rosas C, et al. Crecimiento somático, coordinación motora y actividad física en escolares del Nivel Primario: Implicancias para la Educación Física, Deporte y Salud. Lima, 2005.

14. Haywood KM, Getchell N. Life Span Motor Development. $4^{a}$ edição. USA: Human Kinetics, 2005.

15. Hirtz P, Schielke E. O Desenvolvimento das Capacidades Coordenativas nas Crianças, nos Adolescentes e nos Jovens Adultos. Horizonte 1986;(3):83-88.

\section{AGRADECIMENTOS}

Direção Regional do Desporto da Região Autónoma dos Açores. Faculdade de Desporto da Universidade do Porto.

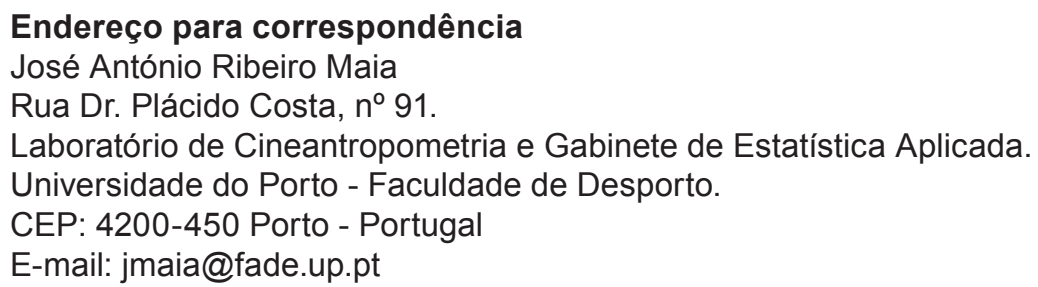

Recebido em 23/10/07 Revisado em 22/11/07 Aprovado em 26/02/08 